\title{
Robust Guaranteed Cost Control of PMSM Chaotic System with Uncertain Parameters
}

\author{
Chuansheng Tang ${ }^{1, *}$, Li Bai ${ }^{1}$, Gang Zhang' ${ }^{2}$, Jie Yang ${ }^{3}$ and Tao $\mathrm{Li}^{4}$ \\ ${ }^{1}$ Nanyang Institute of Technology, Nanyang Henan 473004, China \\ ${ }^{2}$ Nanyang Explosion Proof Electrical Research Institute, Nanyang Henan 473004, China \\ ${ }^{3}$ Henan Institute of Technology, Xinxiang Henan 453000, China \\ ${ }^{4}$ Department of Informatics, University of Zurich, Zurich 8050, Switzerland
}

Received 16 February 2020; Accepted 2 May 2020

\begin{abstract}
A permanent magnet synchronous motor (PMSM) servo system is a multivariable, strong coupling, and complex nonlinear system. Thus, changing the system parameters and control gains can reduce the control performance of the system. To effectively restrain the influence of parameter perturbation on the system performance, a performance control method of PMSM was presented in this study. The method based on the nonlinear dynamic model of PMSM was employed to analyze the chaotic characteristics of bifurcation diagram, attractor, power spectrum, and Lyapunov exponent. The uncertainty model of the system was constructed, two control methods of robust guaranteed performance and robust optimal guaranteed performance were designed, and the effectiveness of the proposed method was verified by simulation experiments. Results demonstrate that, on the one hand, the two designed control methods can quickly switch the system balance point, and the guaranteed performance control method is approximately $1.5 \mathrm{~s}$. Furthermore, the optimal guaranteed performance control is within $1 \mathrm{~s}$, and the optimal guaranteed performance control can ensure the minimum secondary performance index, that is, the minimum control energy is required. On the other hand, the two designed methods have a strong robust performance to the parameter uncertainty of the system. The system parameters have $30 \%$ random perturbation, but the designed methods can still achieve system control effectively. The proposed methods provide certain references for the analysis and control of the machining accuracy of a high-performance servo system.
\end{abstract}

Keywords: PMSM, Chaos control, Robust guaranteed cost control, Secondary performance index, Parameter uncertainty

\section{Introduction}

Permanent magnet synchronous motor (PMSM) and brushless direct current (DC) motor are the two most common types of motors in modern AC servo systems. The PMSM with permanent magnets replaces the excitation winding in the rotor of a wound synchronous motor. The excitation coil, slip ring, and brush are omitted, and the electronic commutation is employed to conduct the operation without brush, which effectively avoids mechanical wear and sparks caused by the mechanical commutation sliding contact, thereby reducing the failure rate, improving the reliability, lengthening the service life, and facilitating maintenance work. The PMSM mainly consists of a stator and a rotor. The rotor is made of a highperformance and affordable permanent magnet material, which enables the motor to have high power density and efficiency. The servo system composed of PMSM is not only employed in the typical equipment of manufacturing fields, such as computer numerical control machine tools and industrial robots, but is also adopted in the automatic aiming and tracking control of radar antenna, aiming control of missile launcher, automatic control of torpedo, disk drive control, roll reduction position control of rolling mill,

*E-mail address: tcsl11@163.com

ISSN: 1791-2377 @ 2020 School of Science, IHU. All rights reserved.

doi:10.25103/jestr.133.20 elevator lifting control, power generation system, solar energy system, and other purposes [1-2].

With the development power electronic, cutting, sensor, and digital technologies, the motor drive system is developing in the direction of high-speed, high-precision, intelligent, and direct drive. However, the servo system composed of PMSM is a complex nonlinear system, and a strong coupling relationship exists among the internal current, flux, speed, and other state variables. In the complex working environment, motor parameters change with temperature, humidity and other factors. The study shows that when the parameters of the motor meet the condition of Hough bifurcation, complex nonlinear phenomena such as limit cycle and chaos occur, accompanied by violent oscillation of speed or torque, irregular electromagnetic noise, and other chaos phenomena [3-4]. This condition seriously reduces the system performance in the highprecision servo situation and may even cause a system collapse.

How to effectively suppress the change of motor parameters and avoid or suppress chaos is a key concern of scholars [5-8], but some problems remain such as poor control performance, insufficient system parameter perturbation suppression ability, or complex controller structure, which causes difficulty in physical realization, especially when the system parameters and control gain change at the same time, the system performance deteriorates, which is the key factor in restricting the 
application of the controller. Based on the analysis of the nonlinear term in the system model and the quadratic optimal performance index, the robust optimal control theory is employed to design a control method that can not only satisfy the quadratic optimal performance index but also have strong interference suppression ability to address the uncertainty of the system model and gain parameters. This study can perfect the motor chaos control theory and improve the design so that it can meet the requirements of practical application.

\section{State of the art}

Since the 1990s, chaos has also been found in motor drives, and study on this area has gradually advanced to include DC motor, induction motor, switched reluctance motor, PMSM, and other drive systems [9]. In recent years, many methods have been employed to realize the chaos control of motor systems. PID control is widely employed in linear system control because of its simple structure and easy physical realization. Karthikeyan et al. [10] applied PI control to the chaos system of permanent magnet DC motor, which achieved good control performance under the condition of system parameter determination and external disturbance free. However, the motor parameters changed with the environment temperature and other factors, thereby causing difficulties in meeting the requirements of high-performance control. Ranjbar et al. [11] combined fuzzy control and PI control for chaos stabilization of induction motors. Although the study compensated for the disturbance caused by uncertain factors of the system in real time by fuzzy control, the fuzzy output of the method was based on the speed and change rate of the error, forming 49 fuzzy rules. The number of rules directly determined the performance of the control system. If the rules are extremely few, then restraining the parameter perturbation becomes difficult. If the rules are excessive, meeting the requirements of fast dynamic response becomes difficult and time consuming. To address these issues, Liu et al. [12] proposed a simple feedback controller with only one state variable on the basis of analyzing the characteristics of the permanent magnet BLDCM chaotic system. Iqbal et al. [13] adopted the method to the chaos control of the permanent magnet synchronous motor; this study had good performance in the deterministic system, but the robust performance was insufficient. Loria [14] proposed a robust linear control method that overcame the load disturbance by combining the load observer with feedback control; the proposed method improved the ability of load disturbance rejection by realtime observation and feedback of external load disturbance, but did not consider the change of system parameters. To overcome the change of system parameters, scholars employed adaptive control [15], sliding mode control [1618], inverse control [19-20], dynamic surface control [21], predictive control [22], finite time control [23], and Lyapunov exponent method [24]. Han [15] overcame the influence of parameter perturbation by online estimation and compensation of uncertain parameters of the system, but online estimation took a long time and affected the dynamic response ability of the system. Sliding mode control was not sensitive to the change of system parameters, and became an effective means to suppress parameter perturbation. Li et al. and Ali et al. [16-17] respectively adopted terminal sliding mode control to control and synchronize permanent magnet synchronous motor, and used a terminal attractor to improve the inherent chattering phenomenon brought by sliding mode control, but chattering was not eliminated, and was closely related to the influence of system uncertainty. In the work of Karthikeyan et al. [18], combined with sliding mode control and adaptive control, which fully utilized the parameter insensitivity of sliding mode control and real-time estimation ability of adaptive control, the chattering of the system was effectively improved, but the complexity of the system was increased and the response ability of the system was reduced. Ye et al. [19] proposed an inverse nonlinear control method for the influence of the parameter uncertainty of the PMSM chaotic system, and employed particle swarm optimization to optimize the control parameters. The proposed method calculated the differentiation of state variables many times, which leaded to the phenomenon of calculation explosion. At the same time, the structure of the controller was complex and difficult to be employed in engineering practice. Karthikeyan et al. [20] combined sliding mode control with inversion control to make full use of their advantages, and employed a robust inversion sliding mode control method. The proposed method was still unable to avoid the computational explosion caused by inversion control. For this reason, Luo et al. [21] introduced low-pass filtering into inversion control to overcome the computational explosion problem caused by differentiation and adopted the adaptive method to estimate and compensate the parameters online. The structure of the controller was complex and the engineering implementation was difficult. The predictive control and Lyapunov index methods proposed by Messadi et al. [22] and Ataei et al. [24] respectively did not consider the influence of the uncertainty of system parameters. Wang et al. [23] introduced a terminal attractor into feedback control, which ensured that the system achieved the desired balance point in a limited time, and had strong antiinterference ability for the change of system parameters. The fractional integral operator was quoted in the proposed method, which was more complex in the circuit implementation and was not conducive to the application in the actual situation.

In addition, the above method does not consider the limitation of control energy in the actual process. In the actual physical system, the output capacity of the controller is always limited, and the control energy required is expected to be as small as possible. Recently, scholars have paid close attention to the optimal control of chaotic systems. Zhu et al. [25] combined linear matrix inequality method and optimal control theory. For the unified chaotic system, a simple optimal controller design method was proposed to avoid the difficulty of solving the nonlinear HamiltonJacobi-Bellman partial differential equation in the method proposed by Marat et al. [26]. Wei et al. [27] extended it to the chaos control of PMSM. Although the aforementioned method considered the secondary performance index of the system, it did not consider the influence of the system parameter uncertainty. In fact, the uncertainty of parameters widely existed in the actual system, and it was more practical to study the optimal control of the chaotic system with parameter uncertainty. Awad et al. [28] proposed an optimal adaptive control and synchronization control strategy for Lorenz chaotic system by combining optimal control with adaptive control. However, the structure of the controller was complex. The introduction of adaptive mechanism increased the cost of the system and reduced its response ability.

The preceding studies mainly focused on the dynamic performance of the PMSM control system, while the study 
on the uncertainty of its control gain is less, especially the study on the limitation of control energy and the uncertainty of system model parameters. Based on the analysis of the nonlinear term in the system model and the quadratic optimal performance index, the robust optimal control theory is employed to design a control method that can not only satisfy the quadratic optimal performance index but also has strong interference suppression ability to the uncertainty of the system model parameters and gain parameters. The study lays a foundation for improving the theory of motor chaos control and aligning the design with the actual needs.

The remainder of this study is organized as follows. Section 3 establishes the PMSM model, analyzes the nonlinear chaos characteristics of the system, completes the design process of robust guaranteed performance control, and proves the stability of the system. Section 4 analyzes the rotor speed and the current characteristic of the model through robust guaranteed performance control method, and obtains the dynamic response curves of rotor speed and current under the controlled system. Section 5 summarizes the conclusions.

\section{Methodology}

\subsection{Chaotic model of PMSM}

The dimensionless mathematical model of PMSM after transformation is as follows [3]:

$$
\left\{\begin{array}{l}
\dot{i}_{d}=-i_{d}+w i_{q}+v_{d} \\
\dot{i}_{q}=-i_{q}-w i_{d}+\gamma w+v_{q} \\
\dot{w}=\sigma\left(i_{q}-w\right)-T_{L}
\end{array}\right.
$$

where $v_{d}$ and $v_{q}$ are the voltages of $d$-axis and $q$-axis after transformation, and $i_{d}$ and $i_{q}$ are the currents of $d$-axis and $q$-axis after transformation, respectively. $w$ and $T_{L}$ are the speed and load after transformation, respectively. $\gamma$ and $\sigma$ are motor parameters.

The chaos phenomenon of the motor is studied when $v_{d}=v_{q}=T_{L}=0$, which is equivalent to the dynamic characteristics of the system under a certain parameter when the motor suddenly loses power after a period of operation. The nonlinear characteristics of the PMSM drive system has been studied on the basis of modern nonlinear theories such as bifurcation and chaos [3]. To effectively understand the complex nonlinear characteristics of the system in a certain range of parameters, the bifurcation diagram of the system state $w$ with changing of the system parameter $\gamma$ is given, as shown in Fig. 1(a). Fig. 1(b) presents a typical chaotic attractor when system parameter $\gamma$ is 26 , system parameter $\sigma$ is 5.46 , and initial state $\left(i_{d 0}, i_{q 0}, w_{0}\right)$ is $(0.01,0.01,0.01)$, in which $i_{d}, i_{q}$, and ware all unit values.

Lyapunov exponent and power spectrum are two effective methods to distinguish a continuous system from a chaotic system. A $3 \mathrm{D}$ continuous system is chaotic if a positive Lyapunov exponent exists. Chaos has a wide and continuous power spectrum, but this power spectrum has many wave peaks, which correspond to the average period of each region of the chaos attractor. Fig. 1 (c) and (d) show the Lyapunov exponents and power spectra, respectively, when $\gamma=26$ and $\sigma=5.46$.

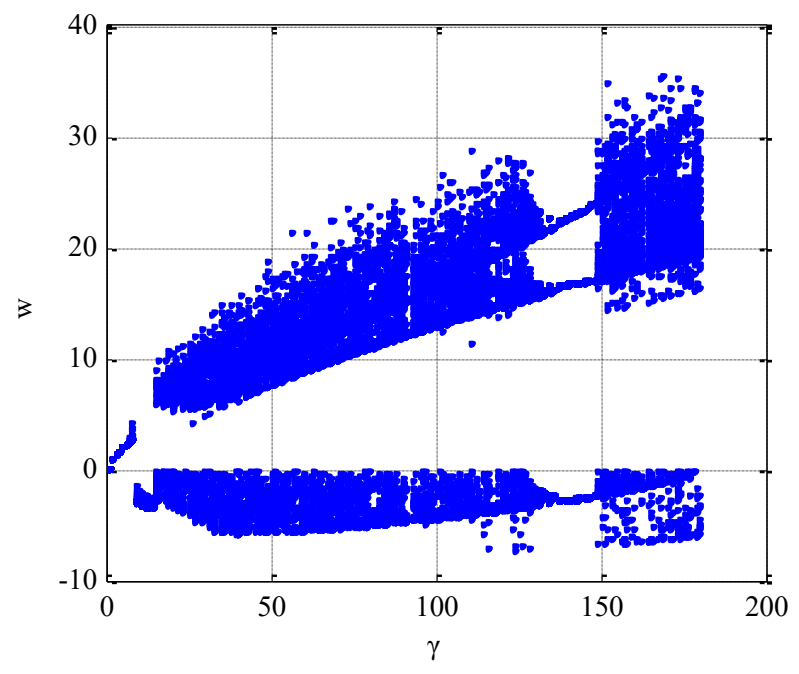

(a) Bifurcation diagram of state variable $w$ with parameter $\gamma$

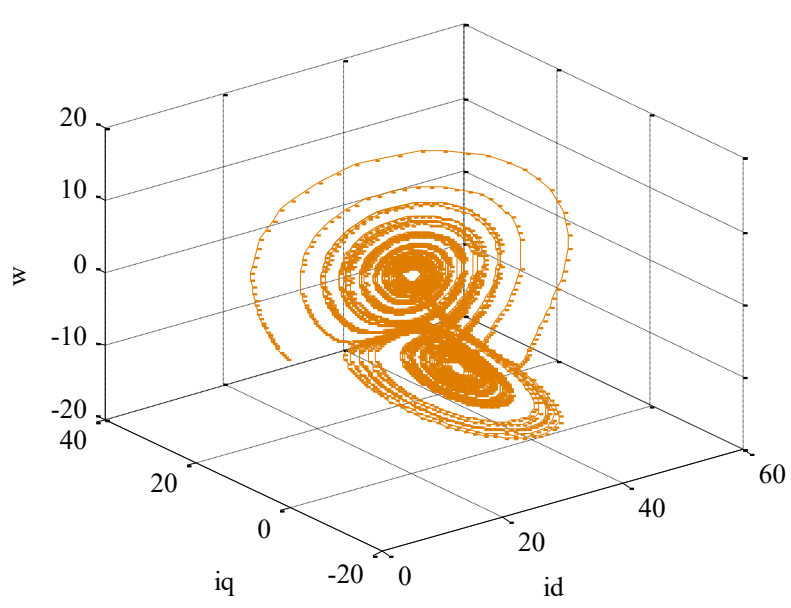

(b) Typical chaotic attractor

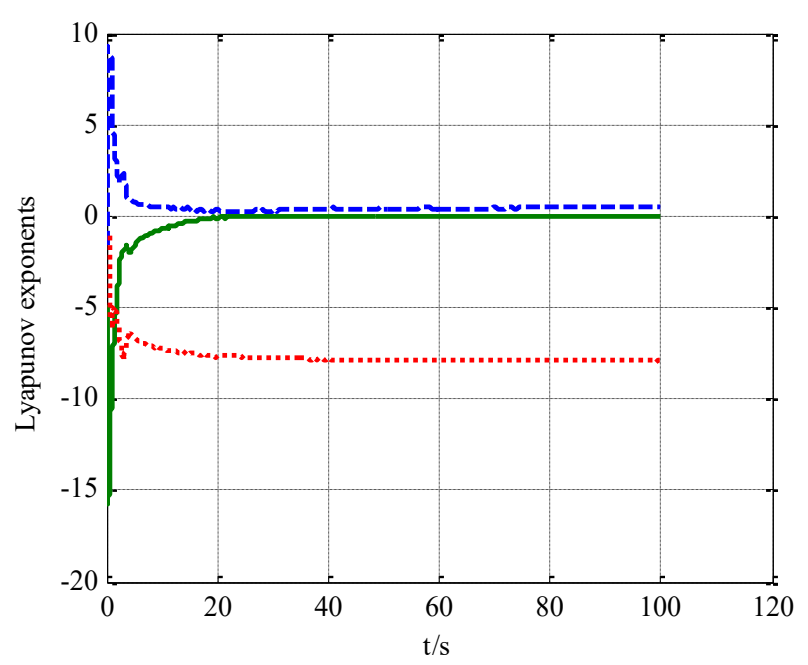

(c) Lyapunov index 


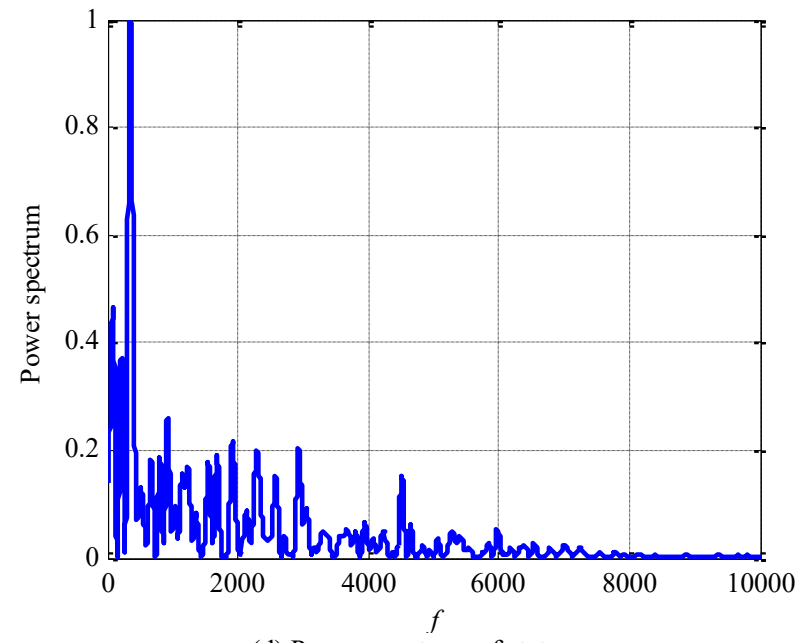

(d) Power spectrum of state $w$

Fig. 1. bifurcation diagram and chaos characteristics of PMSM

Considering the uncertainty of system parameters, the model of controlled PMSM driving system can be expressed as follows:

$$
\left\{\begin{array}{l}
\dot{i_{d}}=-i_{d}+w i_{q}+\left(1+\Delta_{1}\right) u_{1} \\
\dot{i_{q}}=-i_{q}-w i_{d}+\left(\gamma+\Delta_{\gamma}\right) w+\left(1+\Delta_{1}\right) u_{2} \\
\dot{w}=\left(\sigma+\Delta_{\sigma}\right)\left(i_{q}-w\right)
\end{array}\right.
$$

where $\Delta_{\gamma}, \Delta_{\sigma}, \Delta_{1}$, and $\Delta_{2}$ represent the uncertainty of system parameters and control inputs, respectively, which are all boundaries. $u_{1}$ and $u_{2}$ are the control inputs. According to the actual operation of the system, this study assumes that the fluctuation range of system parameters is $30 \%$, that is, $\left\|\Delta_{\gamma}\right\| \leq \delta_{1}=0.3 \gamma,\left\|\Delta_{\sigma}\right\| \leq \delta_{2}=0.3 \sigma,\left\|\Delta_{1}\right\| \leq 0.3$, and $\left\|\Delta_{2}\right\| \leq 0.3$.

Eq. (1) has three equilibrium points, which are $S_{1}(0,0,0) \quad$ and $\quad S_{2,3}(\gamma-1, \pm \sqrt{\gamma-1}, \pm \sqrt{\gamma-1})$ $S_{1}(0,0,0)$ and $S_{2,3}(\gamma-1, \pm \sqrt{\gamma-1}, \pm \sqrt{\gamma-1})$ are the unstable saddle point and focal point, respectively. $S_{1}(0,0,0)$ is the desired equilibrium point, and the non-zero equilibrium point $S_{2,3}(\gamma-1, \pm \sqrt{\gamma-1}, \pm \sqrt{\gamma-1})$ can be transformed into zero equilibrium point by coordinate transformation.

To facilitate the design of the following controllers, Eq. (2) is expressed in an appropriate form as follows:

$\dot{x}=\left(A_{0}+\Delta A\right) x+g(x)+\left(B_{0}+\Delta B\right) u$

where $\quad x=\left(x_{1}, x_{2}, x_{3}\right)^{T}=\left(i_{d}, i_{q}, \omega\right)^{T} \quad, \quad u=\left(u_{1}, u_{2}\right)^{T}$ $A_{0}=\left[\begin{array}{ccc}-1 & 0 & 0 \\ 0 & -1 & \gamma \\ 0 & \sigma & -\sigma\end{array}\right], \Delta A=\left[\begin{array}{ccc}0 & 0 & 0 \\ 0 & 0 & \Delta \gamma \\ 0 & \Delta \sigma & \Delta \sigma\end{array}\right], B_{0}=\left[\begin{array}{ll}1 & 0 \\ 0 & 1 \\ 0 & 0\end{array}\right]$, $\Delta B=\left[\begin{array}{cc}\Delta_{1} & 0 \\ 0 & \Delta_{2} \\ 0 & 0\end{array}\right]$, and $g(x)=\left[\begin{array}{c}x_{2} x_{3} \\ -x_{1} x_{3} \\ 0\end{array}\right]$. (3) is

$$
J(x(t), u(t))=\int_{0}^{\infty}(x(t) Q x(t)+u(r) R u(t)) d t
$$

where $Q$ and $R$ are weighted matrices and positive definite, respectively.

The goal of system design is to determine the gain of the controller $u$ so that Eq. (3) can quickly stabilize to the desired equilibrium point $S_{0}$ from any initial state, and the controller can meet the given performance index Eq. (4) to minimize.

\subsection{Controller design}

To ensure that the designed control is widely used, a class of nonlinear system with the same structure as Eq. (4) can be expressed as follows:

$$
\dot{x}=\left(A_{0}+\Delta A\right) x+g(x)+\left(B_{0}+\Delta B\right) u, x(0)=x_{0}
$$

where $x \in R^{n}$ and $u \in R^{m}$ are the state vector and control input vector of the system, respectively. $A_{0} \in R^{n \times n}$ and $B \in R^{n \times m}$ are the state matrix and control matrix of the system, respectively, in which $m \leq n . x_{0}$ is the initial state. $g(x), \Delta A$, and $\Delta B$ are the nonlinear term and parameter uncertainty matrix of the system, respectively, which satisfy the following assumptions.

Assumption 1 (uncertainty condition). The uncertainty of system parameter $\Delta A$ and change of control gain $\Delta B$ meet the following conditions:

$\left[\begin{array}{ll}\Delta A & \Delta B\end{array}\right]=D F(t)\left[\begin{array}{ll}E_{1} & E_{2}\end{array}\right]$

where $D, E_{1}$, and $E_{2}$ are constant matrices, and $F(t)$ satisfies:

$F^{T}(t) F(t) \leq I, \forall t$

Assumption 2 (nonlinear condition). The nonlinear term of the system $g(x)$ satisfies:

$$
\lim _{x \rightarrow 0} \frac{\|g(x)\|}{\|x\|}=0,\left.g(x)\right|_{x=0}=0
$$

In fact, many chaotic and hyperchaotic systems satisfy nonlinear conditions, such as Chen, Liu, Lü, Lorenz systems and hyperchaotic systems derived from these systems.

Definition [29] A control law $u^{*}(t)$ and an integer $J^{*}$ exist for system Eq. (5) and performance index Eq. (4), so that the closed-loop system is asymptotically stable for all the allowed uncertainties, and the closed-loop performance index satisfies $J \leq J^{*}=\operatorname{tr}(P)$ (trace of matrix P), in which $J^{*}$ is called a performance upper bound of uncertain system Eq. (5), and $u^{*}(t)$ is called a guaranteed performance control law of uncertain system Eq. (5).

The following theorem provides the existence conditions of robust guaranteed cost control law for uncertain nonlinear system Eq. (5). 
Theorem 1 For uncertain nonlinear system Eq. (5) and performance index Eq. (4), if symmetric positive definite matrix $P$, matrix $K$ and normal number $\varepsilon$ all satisfy the uncertain condition Eq. (6) and nonlinear condition Eq. (8), the following equation can be obtained:

$$
A^{T} P+P A+\varepsilon P D D^{T} P+\varepsilon^{-1} E^{T} E+Q+K^{T} R K+M<0
$$

where $u=-K x$ is a guaranteed cost control law of system Eq. (5), and its corresponding upper bound of performance is $J^{*}=\operatorname{tr}(P)$.

Proof. If symmetric positive definite matrix $P$, matrix $K$, and normal number $\varepsilon$ all satisfy the system parameters of uncertain condition Eq. (6), then the matrix inequality Eq. (9) holds. If $u=-K x$, then the corresponding closed-loop system is

$$
\dot{x}=\left[A_{0}-B_{0} K+D F\left(E_{1}-E_{2} K\right)\right] x+g(x), x(0)=x_{0}
$$

Selecting Lyapunov function $V=x^{T} P x$ is derived along the closed-loop system Eq. (10), and the following expressions can be obtained:

$$
\begin{aligned}
\dot{V}= & \dot{x}^{T} P x+x^{T} P \dot{x} \\
= & {[(A+\Delta A-\Delta B K) x+g(x)]^{T} P x+} \\
& x^{T} P[(A+\Delta A-\Delta B K) x+g(x)]
\end{aligned}
$$

Furthermore,

$$
\begin{aligned}
& (\Delta A-\Delta B K)^{T} P+P(\Delta A-\Delta B K) \\
& =\left[D F(t)\left(E_{1}-E_{2} K\right)\right]^{T} P+P D F(t)\left(E_{1}-E_{2} K\right) \\
& \leq \varepsilon P D D^{T} P+\varepsilon^{-1} E^{T} E
\end{aligned}
$$

Thus, the following equations can be obtained:

$$
\begin{aligned}
\dot{V} & \leq x^{T}\left[A^{T} P+P A^{T}+\varepsilon P D D^{T} P+\varepsilon^{-1} E^{T} E\right] x+2 x^{T} P g(x) \\
& =x^{T}\left[-Q-K^{T} R K-M\right] x+2 x^{T} P g(x) \\
& <x^{T}\left[-Q-K^{T} R K\right] x-V_{1}
\end{aligned}
$$

where $V_{1}=x^{T} M x-2 x^{T} \operatorname{Pg}(x)$.

As the system satisfies the nonlinear condition Eq. (8), that is, $\lim _{x \rightarrow 0} \frac{\|g(x)\|}{\|x\|}=0$, there is $\forall \xi$ and $\exists \delta>0$. When $\|x\| \leq \delta$, there is $\frac{\|g(x)\|}{\|x\|}<\xi$, so $\|g(x)\|<\xi\|x\|$ can be obtained.

Then, the following equations can be obtained:

$$
\begin{aligned}
& \begin{aligned}
2 x^{T} P g(x) & \leq 2\left\|x^{T} P\right\|\|g(x)\|=2 \sqrt{x^{T} P P x}\|g(x)\| \\
& \leq 2 \sqrt{\lambda_{\max }\left(P^{2}\right)}\|x\|\|g(x)\|
\end{aligned} \\
& x^{T} M x \leq \lambda_{\min }(M)\|x\|^{2}
\end{aligned}
$$

where $\lambda_{\min }(*)$ and $\lambda_{\max }(*)$ represent the minimum and maximum eigenvalues of the matrix $*$, respectively. $P$ and $M$ are symmetric positive definite matrix, so $\lambda_{\text {min }}(M)>0$ and $\lambda_{\text {min }}\left(P^{2}\right)>0$.

Then, when $\|x\| \leq \delta$, the following equation can be obtained:

$V_{1}=x^{T} M x-2 x^{T} P g(x)<-\left[\lambda_{\min }(M)-2 \xi \sqrt{\lambda_{\min }\left(P^{2}\right)}\right]\|x\|^{2}$

As $\lambda_{\min }(M)$ and $\lambda_{\min }\left(P^{2}\right)$ are definite quantities, a positive number $\varepsilon$ exists, and $V_{1}<0$ when $\xi<\frac{\lambda_{\min }(M)}{2 \lambda_{\min }\left(P^{2}\right)}$. Thus, the following equations can be obtained:

$\dot{V}<x^{T}\left[-Q-K^{T} R K\right] x<0$.

Let us prove $J \leq J^{*}=\operatorname{tr}(P)$.

The two sides of Eq. (16) are integrated, and the time $t$ is from zero to infinity. When $t \rightarrow \infty, V(x(t)) \rightarrow 0$. Therefore, $J \leq E\left\{V(x(0)\}=E\left\{x_{0}{ }^{T} P x_{0}\right\}=\operatorname{tr}(P)=J *\right.$ can be obtained.

Thus, theorem 1 is proved.

Theorem 2 For uncertain nonlinear systems Eq. (5) and performance indexes Eq. (4), if symmetric positive definite matrices $X=X^{T}=P^{-1}$, matrices $Y=K X$, and normal number $\varepsilon$ exist, then the following matrix inequalities are true for all the uncertain conditions Eq. (6) and nonlinear conditions Eq. (8):

$$
\left[\begin{array}{cccc}
\Xi & X E_{1}^{T}-Y^{T} E_{2}^{T} & X & Y^{T} \\
* & -\varepsilon I & 0 & 0 \\
* & * & -(Q+M)^{-1} & 0 \\
* & * & * & -R^{-1}
\end{array}\right]<0
$$

where $\quad \Xi=A_{0} X+X A_{0}^{T}-B_{0} Y-Y^{T} B_{0}^{T}+\varepsilon D D^{T}$, and * represents the transposition of the corresponding elements in the matrix. $u=-K x=-Y X^{-1} x$ is a guaranteed cost-control law of system Eq. (5), and its corresponding upper bound of performance is $J^{*}=\operatorname{tr}(P)$.

Proof. $X=X^{T}=P^{-1}$ and $Y=K X$ are substituted into Eq. (17). Then, Eq. (17) and (18) are essentially equivalent by using Schur complement lemma. Therefore, its proof process can refer to the proof of Theorem 1 .

Theorem 2 only transforms matrix inequality Eq. (17) into a form that is easy to solve by linear matrix inequality (LMI).

Theorems 1 and 2 show that the upper bound of the system performance depends on the selection of guaranteed cost control law. The issues are how to choose an appropriate guaranteed cost control law to minimize the upper bound of system performance. This is the optimal guaranteed cost-control problem of Eq. (5). The control law that makes the upper bound of Eq. (5) performance minimum is called optimal guaranteed cost-control law.

Theorem 3 For uncertain nonlinear system Eq. (5) and performance index Eq. (4), the following optimization problem is 
$\min _{\varepsilon, a, X, Y} \operatorname{Trace}(a)$

$\left[\begin{array}{cccc}\Xi & X E_{1}^{T}-Y^{T} E_{2}^{T} & X & Y^{T} \\ * & -\varepsilon I & 0 & 0 \\ * & * & -(Q+M)^{-1} & 0 \\ * & * & * & -R^{-1}\end{array}\right]<0$

$\left[\begin{array}{cc}\alpha & x_{0}^{T} \\ x_{0} & X\end{array}\right]>0$

If a solution $(\varepsilon, \alpha, X, Y)$ exists, then $u=-K x=-Y X^{-1} x$ is the optimal guaranteed cost control law of Eq. (5), and its corresponding minimum performance upper bound is $\alpha$.

Proof. From Theorem 2, Eq. (20) can guarantee that $u=-K x=-Y X^{-1} x$ is a guaranteed performance control law of Eq. (5), and its corresponding upper bound of performance is $J^{*}=\operatorname{tr}(P)$.

Schur's complement lemma shows that Eq. (21) is equivalent to $\alpha>x_{0}^{T} X^{-1} x_{0}=x_{0}^{T} P x_{0}$, the minimization of $\operatorname{Trace}(\alpha)$ guarantees the minimization of $\operatorname{Trace}(P)$ and the minimization of the upper bound of system performance. The objective function and constraint function in Eq. (19) are convex functions of variables. Eq. (19) is a convex optimization problem; thus, it has a global minimal value.

Thus, theorem 3 is proved.

\section{Results of analysis and discussion}

This part mainly employs digital simulation of PMSM chaotic system to validate the proposed theorem in Section 3. First, this study verifies that the PMSM chaotic system Eq. (3) satisfies the uncertainty condition Eq. (6) and nonlinear condition Eq. (8).

The following equations can be obtained by assumption 1 in Section 3.2 as follows:

$\left[\begin{array}{ll}\Delta A & \Delta B\end{array}\right]=D F(t)\left[\begin{array}{ll}E_{1} & E_{2}\end{array}\right], F^{T}(t) F(t) \leq I, \forall t$

where $\quad F(t)=\operatorname{diag}(\operatorname{rand}(1), \operatorname{rand}(1), \operatorname{rand}(1))$

$D=\operatorname{diag}(0,0.3,0.3), E_{1}=\left[\begin{array}{ccc}0 & 0 & 0 \\ 0 & 0 & \gamma \\ 0 & \sigma & -\sigma\end{array}\right]$, and $E_{2}=\left[\begin{array}{cc}1 & 0 \\ 0 & 1 \\ 0 & 0\end{array}\right]$.

Obviously, Eq. (22) satisfies the uncertainty condition Eq. (6).

$$
\begin{aligned}
\lim _{x \rightarrow 0} \frac{\|g(x)\|}{\|x\|}= & \lim _{e \rightarrow 0} \frac{\sqrt{x_{2}^{2} x_{3}^{2}+x_{1}^{2} x_{3}^{2}}}{\sqrt{x_{1}^{2}+x_{2}^{2}+x_{3}^{2}}} \\
& \leq \lim _{x \rightarrow 0} \frac{\sqrt{x_{2}^{2} x_{3}^{2}+x_{1}^{2} x_{3}^{2}}}{\sqrt{x_{1}^{2}}}=\lim _{x \rightarrow 0} \sqrt{x_{3}^{2}+x_{3}^{2}}=0
\end{aligned}
$$

where $\left.g(x)\right|_{x=0}=0$, that is, Eq. (23) also satisfies the nonlinear condition Eq. (7), so the controller gain can be solved by using the theorems in Section 3.2.
The MATLAB platform is divided into three cases to simulate the PMSM chaotic system. The initial state of the system is $S_{0}=(0.01,0.01,0.01)$, the expected equilibrium point is $S_{d}=S_{2}(25,5,5)$ when $t=20 \mathrm{~s}$, and the expected equilibrium point is $S_{d}=S_{2}(25,-5,-5)$ when $t=40 \mathrm{~s}$.

\subsection{Fixed parameters in system model}

When the parameters in the system model is fixed, the simulation results of optimal control (OC), guaranteed cost control (GCC), and optimal guaranteed cost control (OGCC) are shown in Fig. 2.

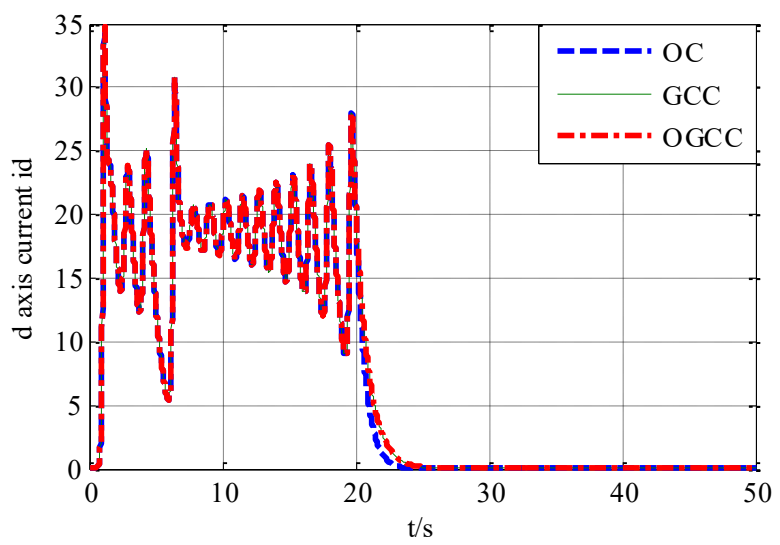

(a) $d$-axis current response curve

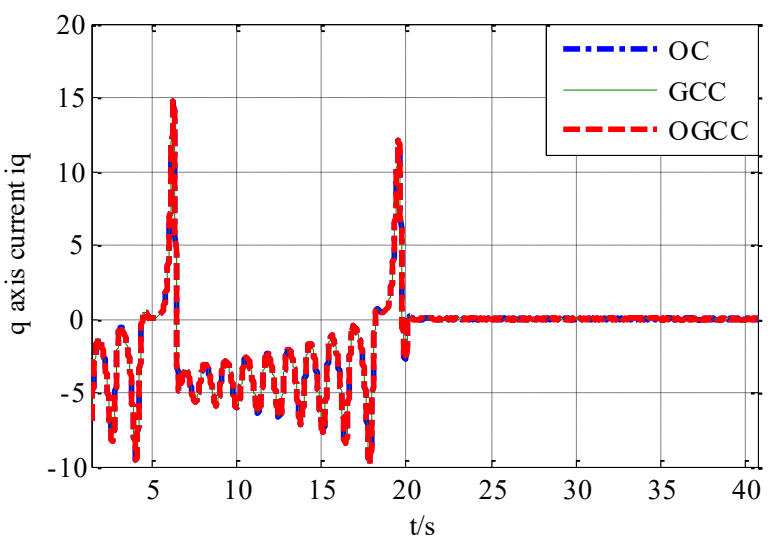

(b) $q$-axis current response curve

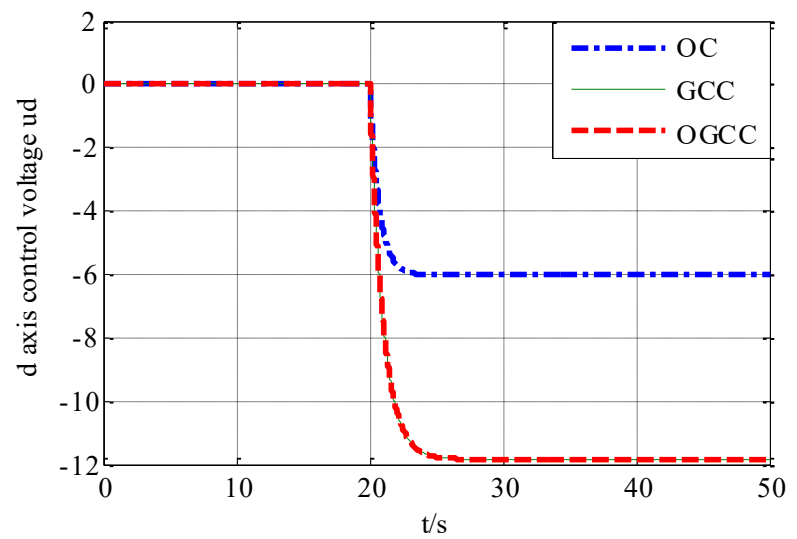

(c) $d$-axis voltage control curve 


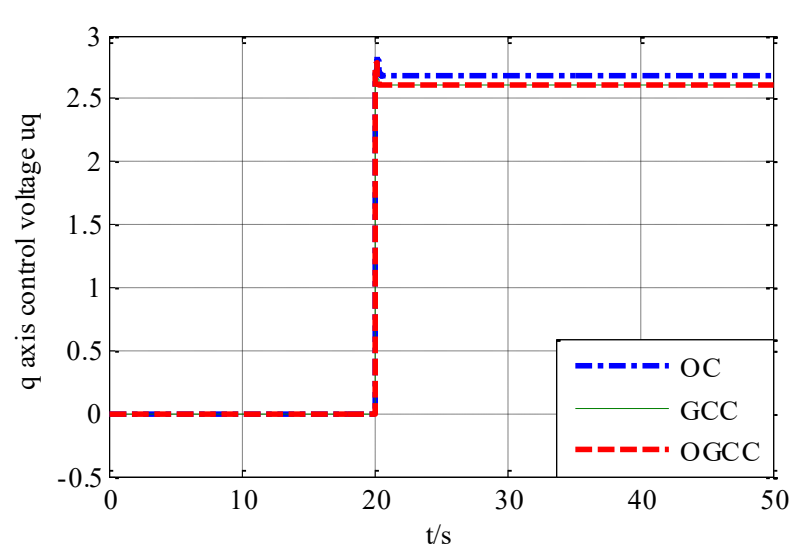

(d) $q$-axis voltage control curve

Fig. 2. Response curve of system without parameter perturbation

Fig. 2 shows that the parameter change and control gain perturbation in the system are both zero, that is, $\left[\begin{array}{ll}\Delta A & \Delta B\end{array}\right]=\left[\begin{array}{ll}0 & 0\end{array}\right]$. When the optimal control, guaranteed cost control, and optimal guaranteed cost control are adopted, the following results are obtained, respectively:

$$
\begin{aligned}
& P_{1}=\left[\begin{array}{ccc}
0.4142 & 0 & 0 \\
0 & 15.1612 & 23.4602 \\
0 & 23.4602 & 37.3651
\end{array}\right] \\
& P_{2}=\left[\begin{array}{ccc}
1.3599 & 0 & 0 \\
0 & 51.2436 & 51.8733 \\
0 & 51.8733 & 78.7125
\end{array}\right] \\
& P_{3}=\left[\begin{array}{ccc}
0.8425 & -0.0005 & 0.0001 \\
-0.0005 & 23.4373 & 23.0301 \\
0.0001 & 23.0301 & 39.8521
\end{array}\right]
\end{aligned}
$$

Fig. 2 shows that the three methods can quickly approach the expected equilibrium point when the model parameters in the system are all determined, the corresponding performance indexes of optimal control, guaranteed cost control, and optimal guaranteed cost control are, respectively, $J_{1}^{*}=0.0192 \quad, \quad J_{2}^{*}=0.0235$ and $J_{3}^{*}=0.0110$. The optimal guaranteed cost control has the best performance index.

\subsection{Uncertainty of system parameters and control gains in system model}

The simulation results of optimal control, guaranteed cost control, and optimal guaranteed cost control are presented in Fig. 3 when system parameters and control gains in the system model are uncertain.

Fig. 3 shows that the parameter variation and control gain perturbation in the system include 30\% parameter perturbation.

When the optimal control, guaranteed cost control, and optimal guaranteed cost control are adopted, the following results are obtained respectively:

$$
\begin{aligned}
& P_{1}=\left[\begin{array}{ccc}
0.4142 & 0 & 0 \\
0 & 15.1612 & 23.4602 \\
0 & 23.4602 & 37.3651
\end{array}\right] \\
& P_{2}=\left[\begin{array}{ccc}
1.2254 & 0 & 0 \\
0 & 37.0011 & 60.2247 \\
0 & 60.2247 & 136.1788
\end{array}\right] \\
& P_{3}=\left[\begin{array}{ccc}
0.7441 & -0.0001 & -0.0003 \\
-0.0001 & 27.0656 & 48.2589 \\
-0.0003 & 48.2589 & 114.1418
\end{array}\right]
\end{aligned}
$$

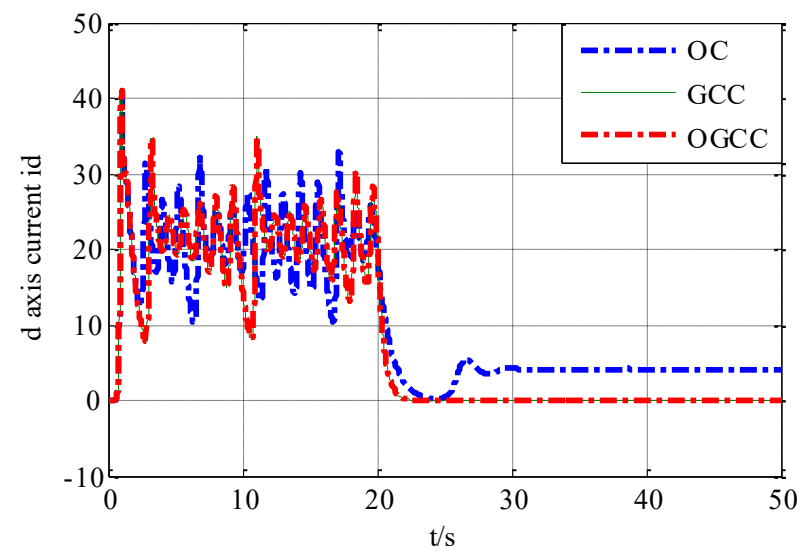

(a) $d$-axis current response curve

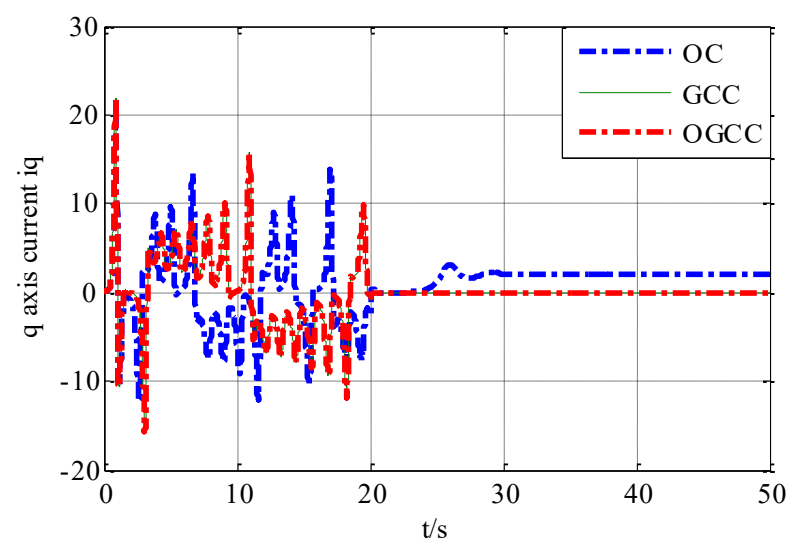

(b) $q$-axis current response curve

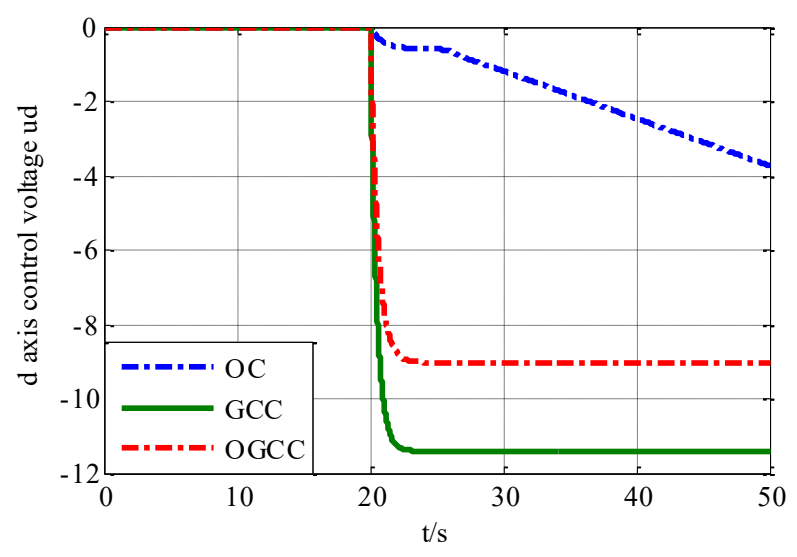

(c) $d$-axis voltage control curve 


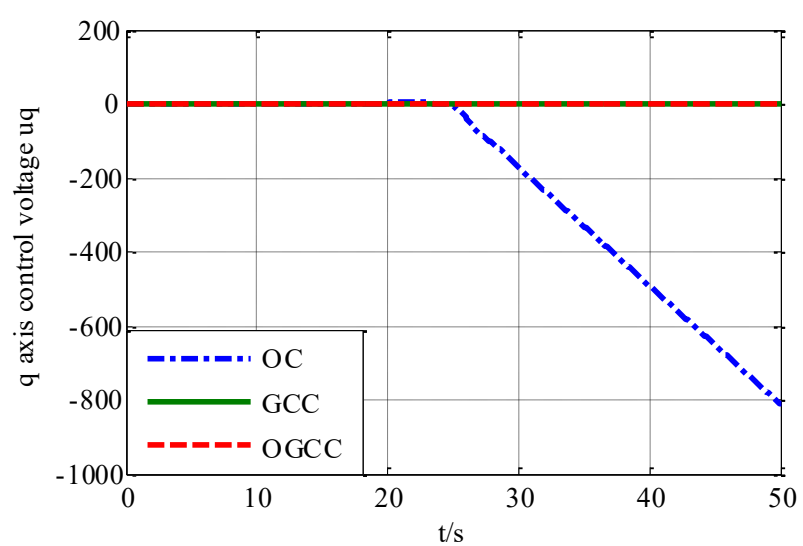

(d) $q$-axis voltage control curve

Fig. 3. Response curve of system with parameter uncertainty

Fig. 3 shows that model parameters and control gains are uncertain in the system, and the following conclusions can be drawn: (1) Guaranteed cost control and optimal guaranteed cost control can still guarantee the system to stabilize rapidly to the desired equilibrium point $S_{1}(0,0,0)$. Guaranteed cost control method is approximately $1.5 \mathrm{~s}$, whereas the optimal guaranteed cost control is within $1 s$, and the optimal control system deviates from the equilibrium point because it cannot compensate for the influence of uncertainty. (2) The corresponding performance indexes of guaranteed cost control and optimal guaranteed cost control are $J_{2}^{*}=0.0295$ and $J_{3}^{*}=0.0238$, respectively. The uncertainty of model parameters and control gains can increase the performance index, but the optimal guaranteed cost control still has a smaller index value than the guaranteed cost control.

\subsection{Switch tracking control among different system equilibrium points}

The system parameters and gain system are switched at different equilibrium points, and results of simulation are shown in Fig. 4.

According to theorems 2 and 3, the guaranteed cost control law and optimal guaranteed cost control law of PMSM chaotic system Eq. (3) are obtained, respectively, and the initial point of the system is $(1,0,-1)$. To compare the performance of the guaranteed cost control and optimal guaranteed cost control, the control parameters are selected as follows:

$\varepsilon=0.8, R=I^{2 \times 2}, Q=M=I^{3 \times 3}$

(1) Stable to equilibrium point $S_{1}(0,0,0)$

According to theorem 2, the feedback gain and performance index of the guaranteed cost-control law of the system are obtained as follows:

$K_{1}^{1}=\left[\begin{array}{ccc}0.5804 & 0 & 0 \\ 0 & 29.4649 & 49.5145\end{array}\right], \alpha_{1}^{1}=133.8245$

According to theorem 3, the feedback gain and performance index of the optimal guaranteed cost-control law of the system are obtained as follows:

$$
K_{1}^{2}=\left[\begin{array}{ccc}
0.3831 & 0 & 0 \\
0 & 22.5474 & 43.4810
\end{array}\right], \alpha_{1}^{2}=109.8266
$$

(2) Stable to equilibrium point $S_{2}(25,5,5)$

According to theorem 2, the feedback gain and performance index of the guaranteed cost control law of the system are obtained as follows:

$$
K_{2}^{1}=\left[\begin{array}{ccc}
2.9749 & -0.4448 & 2.9980 \\
-0.4242 & 7.7908 & 17.5754
\end{array}\right], \alpha_{2}^{1}=51.7338
$$

According to theorem 3, the feedback gain and performance index of the optimal guaranteed cost control law of the system are obtained as follows:

$$
K_{2}^{2}=\left[\begin{array}{ccc}
2.0402 & -0.6473 & 1.8547 \\
-0.6473 & 5.2357 & 16.4728
\end{array}\right], \alpha_{2}^{2}=35.8572
$$

(3) Stable to equilibrium point $S_{3}(25,-5,-5)$

According to theorem 2, the feedback gain and performance index of the guaranteed cost control law of the system are obtained as follows:

$$
K_{3}^{1}=\left[\begin{array}{lll}
2.9749 & 0.4448 & -2.9980 \\
0.4242 & 7.7908 & 17.5754
\end{array}\right], \alpha_{3}^{1}=114.8852
$$

According to theorem 3, the feedback gain and performance index of the optimal guaranteed cost control law of the system are obtained as follows:

$$
K_{3}^{2}=\left[\begin{array}{lll}
2.0455 & 0.6464 & -1.8495 \\
0.6464 & 5.2358 & 16.4720
\end{array}\right], \alpha_{3}^{2}=52.5494
$$

Robust guaranteed cost control and optimal guaranteed cost control can quickly stabilize the system to the desired equilibrium point from the aforementioned results and the simulation in Fig. 4, and the change of parameters have strong robustness in the system. Although the robust guaranteed cost control is more able to ensure that the control system meets certain performance indexes than the robust optimal guaranteed cost control, it cannot guarantee the minimum upper bound of the performance indexes. The gain value of the robust guaranteed cost control is larger than the robust optimal guaranteed cost control, which requires further control energy to achieve the control effect. Therefore, the robust optimal guaranteed cost control has a higher application value.

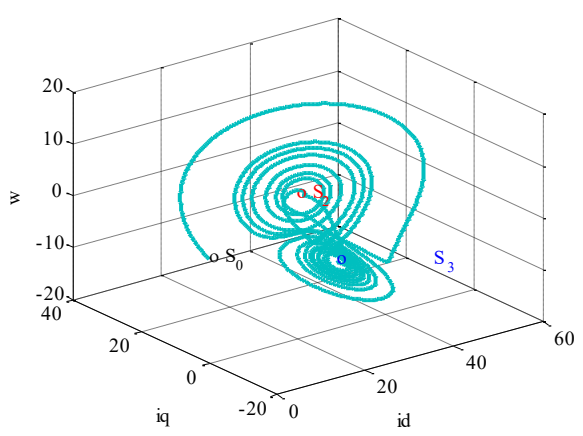

(a) Phase diagram of PMSM chaotic system with guaranteed cost control 


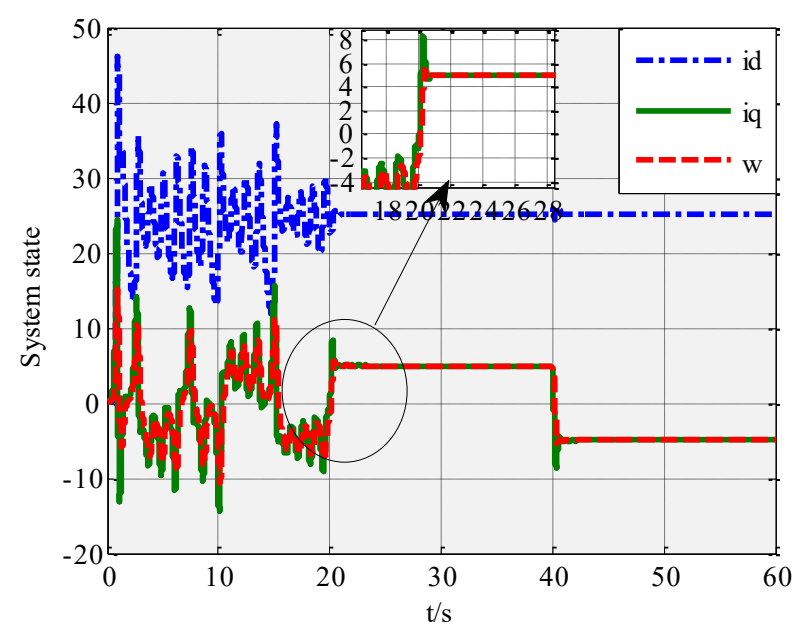

(b) State response curve of PMSM chaotic system with guaranteed cost control

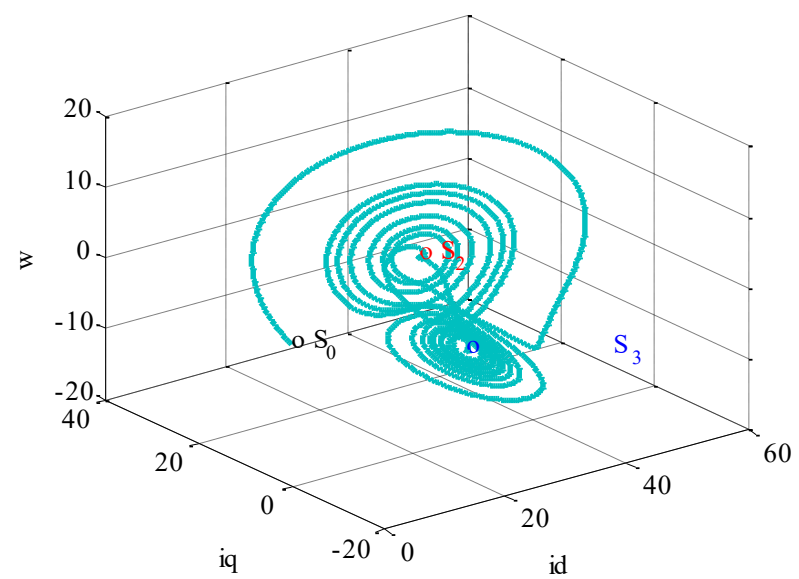

(c) Phase diagram of PMSM chaotic system with robust optimal guaranteed cost control

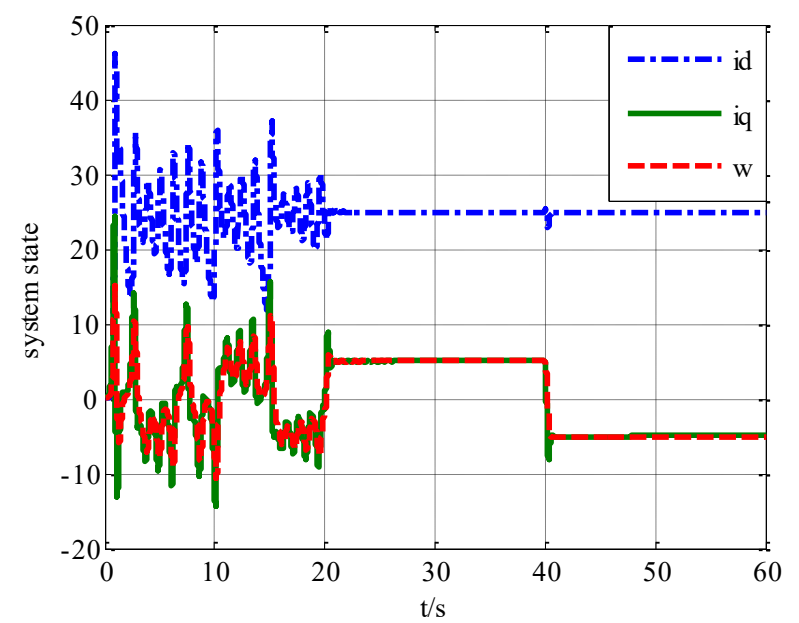

(d) State response curve of PMSM chaotic system with robust optimal guaranteed cost control

Fig. 4. State response curve of system with different switching points

\section{Conclusions}

The characteristics of the nonlinear term system based on the structural characteristics of PMSM were analyzed to suppress the influence of model parameter perturbation and control gain perturbation on the performance of the PMSM chaotic system, and the control performance of the system in combination with the robust optimal control theory was studied. Finally, the following conclusions are drawn:

(1) The optimal control, guaranteed cost control, and optimal guaranteed cost control can effectively stabilize the system state to the desired equilibrium point when the system does not have model parameter perturbation and control gain perturbation, and the optimal guaranteed cost control has the minimum upper bound of the performance index.

(2) The change of system model parameters and control gains cause the optimal control to deviate from the expected equilibrium point, while the guaranteed cost control and optimal guaranteed cost control can still effectively track the equilibrium point. The upper bound of the performance index of the guaranteed cost control and optimal guaranteed cost control is increased, and the dynamic response of the optimal guaranteed cost control is faster.

(3) When different equilibrium points of the system are switched tracking control, both guaranteed cost control and optimal guaranteed cost control can reach the desired equilibrium point quickly, but a slight overshoot in the switching process occurs.

Thus, a robust optimal guaranteed cost-control method is proposed by combining the simulation experiment with theory. The proposed method can effectively overcome the influence of the model parameter perturbation and control gain perturbation of the PMSM chaotic system on the system performance, and the robust optimal guaranteed costcontrol energy can meet certain performance indicators. The proposed method is suitable for the actual working conditions, and is relevant to the later application in the high-performance servo system.

\section{Acknowledgements}

This study was supported by the Key Scientific Study Projects of Higher Education Institutions of Henan Province (Grant Nos. 20B470003 and 18B470007) and the Promotion Special Project of Scientific Study Program of Henan Province (Grant Nos. 202102210298 and 202102210084).

This is an Open Access article distributed under the terms of the Creative Commons Attribution License

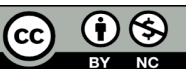

\section{References}

1. Lin, L., He, Y., Zhou, J. H., Chen, H. H., "Research for megawatt direct-driven permanent magnet synchronous wind generator system". Small and Special Electrical Machines, 48(1), 2020, pp. $12-16+20$.

2. Deniz, E., "ANN-based MPPT algorithm for solar PMSM drive system fed by direct-connected PV array". Neural Computing and Applications, 28(10), 2017, pp.3061-3072.
3. Sun, L. X., Lu, S., Wen, Z. G., Li, Y. F., "Analysis of chaotic motion mechanism of permanent magnet synchronous motors". Electric Machines and Control, 23(3), 2019, pp.97-104.

4. Zhang, F. C., Liao, X. F., Mu, C. L., "Dynamical analysis of the permanent-magnet synchronous motor chaotic system". Advances in Difference Equations, (1), 2017, pp.1-10. 
5. Hatem, T., Mohamed, B., "Control of chaos in permanent magnet synchronous motor with parameter uncertainties: a lyapunov approach". International Journal of Innovation and Scientific Research, 13(1), 2015, pp. 279-285.

6. Zhou, P., Cai, H., Yang, C. D., "Stabilization of the unstable equilibrium points of the fractional-order BLDCM chaotic system in the sense of Lyapunov by a single-state variable". Nonlinear Dynamics, 84(4), 2016, pp.2357-2361.

7. Jay, P. S., Binoy, K. R., Nikolay, V. K., "Multistability and hidden attractors in the dynamics of permanent magnet synchronous motor". International Journal of Bifurcation and Chaos, 2017, doi: org/10.1142/S0218127419500561.

8. Luo, S. H., Wang, J.X., Wu, S. L., Xiao, K., "Chaos RBF dynamics surface control of brushless DC motor with time delay based on tangent barrier Lyapunov function". Nonlinear Dynamics, 78(2), 2014, pp.1193-1204.

9. Chau, K. T., Wang, Z., "Design, analysis, and experimentation of chaotic permanent magnet DC motor drives for electric compaction". IEEE Transactions on Circuits and Systems II: Express Briefs, 56(3), 2009, pp.245-249.

10. Karthikeyan, R., Sundarapandian, V., Anitha, K., Prakash, D., "Dynamic analysis and chaos suppression in a fractional order brushless DC motor". Electrical Engineering, 99(2), 2017, pp.721733.

11. Ranjbar, N. A., Kholerdi, H.A., "Chaotification and fuzzy PI control of three-phase induction machine using synchronization approach". Chaos Solitons and Fractals, 91, 2016, pp.443-451.

12. Liu, D., Zhou, G. P., Liao, X. X., "Global exponential stabilization for chaotic brushless DC motor with simpler controllers". Transactions of the Institute of Measurement and Control, 41(9), 2019, pp.2678-2684.

13. Iqbal, A., Singh, G. K., "Chaos control of permanent magnet synchronous motor using simple controllers". Transactions of the Institute of Measurement and Control, 41(8), 2019, pp.2352-2364.

14. Loria, A., "Robust linear control of (chaotic) permanent-magnet synchronous motors with uncertainties". IEEE Transactions on Circuits and Systems I Regular Papers, 56(9), 2009, pp.2109-2122.

15. Han, H. C., "Adaptive control of a chaotic permanent magnet synchronous moto". Nonlinear Dynamics, 69(3), 2012, pp.13111322 .

16. Li, C. L., Wu, L., "Sliding mode control for synchronization of fractional permanent magnet synchronous motors with finite time". Optik-International Journal for Light and Electron Optics, 127(6), 2016, pp. 3329-3332.
17. Ali, N., Rehman, A. U., Alam, W., Maqsood, H., "Disturbance observer based robust sliding mode control of permanent magnet synchronous motor". Journal of Electrical Engineering and Technology, 14(6), 2019, pp.2531-2538.

18. Karthikeyan, R., Fahime, N., Anitha, K., Ashokkumar, S., Sajad, J., "Fractional order synchronous reluctance motor: analysis, chaos control and FPGA Implementation". Asian Journal of Control, 20(5), 2018, pp.1979-1993.

19. Ye, J. H., Yang, J. H., Xie, D. S., Huang, B. Z., Cai, H., "Strong robust and optimal chaos control for permanent magnet linear synchronous Motor". IEEE Access, 7, 2019, pp.57907-57916.

20. Karthikeyan, R., Fahime, N., Anitha, K., Prakash, D., "Dynamic analysis and chaos suppression in a fractional order brushless DC motor". Electrical Engineering, 99(2), 2017, pp.735-735.

21. Luo, S. H., Gao, R. Z., "Chaos control of the permanent magnet synchronous motor with time-varying delay by using adaptive sliding mode control based on DSC". Journal of the Franklin Institute, 355(10), 2018, pp.4147-4163.

22. Messadi, M., Mellit, A., "Control of chaos in an induction motor system with LMI predictive control and experimental circuit validation". Chaos Solitons and Fractals, (97), 2017, pp.51-58.

23. Wang, M. F., Wei, D. Q., Luo, X. S., Zhang, B., "Chaos control in a brushless DC motor based on finite-time stability theory". Journal of Vibration and Shock, 35(13), 2016, pp.90-93.

24. Ataei, M., Kiyoumarsi, A., Ghorbani, B., "Control of chaos in permanent magnet synchronous motor by using optimal Lyapunov exponents placement”. Physics Letters A, 374(41), 2010, pp.42264230.

25. Zhu, S. P., Qian, F. C., Liu, D., "Optimal control for uncertainty dynamic chaotic systems". Acta Physica Sinica, 59(4), 2010, pp.2250-2255.

26. Marat, R., Mamoel, B. J., "On an optimal control for design for Rossler system". Physics Letters A, 333(3), 2004, pp.241-245.

27. Wei, Q., Wang, X. Y., Hu, X. P., "Optimal control for permanent magnet synchronous motor". Journal of Vibration and Control, 20(8), 2014, pp.1176-1184.

28. Awad, E. G., Ammar, S., "Optimal control and synchronization of Lorenz system with complete unknown parameters". Chaos Solitons and Fractals, 30(5), 2006, pp.1122-1132.

29. Yu, L., "Robust control-linear matrix inequalities approach". Beijing: Tsinghua University Press, China, 2002, pp.6-13. 